\title{
ANATOMICAL MEASUREMENTS RELATIVE TO CONDUCTIVE ANESTHESIA WITH PROCAINE AND ALCOHOLIC INJECTION FOR TIC DOULOUREUX.
}

\author{
By Wmiam A. Cook, D. D. S., Detroit, Mich.
}

$\mathrm{U}$ NDER this title are given the findings resultant from a series of measurements and observations on 100 dried adult skulls and mandibles. The skulls and mandibles ineasured are those used in teaching osteology in the Medical Department of the University of Michigan, and are tabulated that they may be readily referred to. On each of the 100 , fifty-two measurements and notations were made.

The main object in compiling these charts was the establishment of facts and landmarks relative to conductive anesthesia with procaine, and alcoholic injection for tic douloureux. And with the exception of the infraorbital the technic in each case is the same for alcoholic injection as for conductive anesthesia.

The measurements which are of greatest interest under this title are those relative to the distribution of the maxillary and mandibular divisions of the fifth or Trigeminal nerve.

All measurements were made with accurate instruments, which included:

(1) A system of revolving, parallel planes and lines, and a triangle of $90^{\circ}$, $45^{\circ}$ and $15^{\circ}$ for the establishment of angles.

(2) A pair of steel dividers (calipers) and

(3) A millimeter ruler.

We will first consider the measurements for determining the location of the mental foramen.
These measurements were made by placing one point of the dividers on the inferior border of the mandible and the other point on the inferior border of the foramen, and measuring the spread of the dividers on a millimeter ruler. The combined notations establish the fact that the foramen is very constant with the inferior border, and by observation it is readily seen that it is very inconstant with the superior border of the mandible. In edentulous jaws the foramen may open immediately on the superior surface (Figure 1) and in others it may be 25 millimeters (Figure 2) to the superior border.

In the 100 mandibles, totaling 200 foramina located, 176 of the foramina fell within the limits of $11 \mathrm{~mm}$. and 15 $\mathrm{mm}$. from the inferior border of the mandible. This shows a variation of only $4 \mathrm{~mm}$. in $7 / 8$ of the cases. Of the remaining 24 foramina, 18 were $16 \mathrm{~mm}$., 3 were $17 \mathrm{~mm}$., 1 was $20 \mathrm{~mm}$., and 2 (in the same mandible) were 9 and 10 $\mathrm{mm}$. respectively, above the inferior border of the mandible.

In making a hypodermic injection for mental conductive anesthesia, the needle should be projected in a line parallel to the bucco-lingual plane of the long axis of the second bicuspid, and in the line of the foramen (slightly anterior to the apex of the second bicuspid). Eliminating the one mandible, the foramina of which measured 9 and 10 $\mathrm{mm}$. respectively, and taking $11 \mathrm{~mm}$. as the minimum distance of the foramen 
above the inferior border of the mandible and $2 \mathrm{~mm}$. as the diameter of the foramen, then in proceeding with the injection from a point slightly anterior to the second bicuspid the foramen will have been found in $100 \%$ of the cases, when the needle has reached a point 13 mm. above the inferior border of the mandible, because all of the foramina above that point will have been found as the needle proceeds in contact with the bone from the point of injection to that point.

To have an accurate technic, the point of injection should be marked by

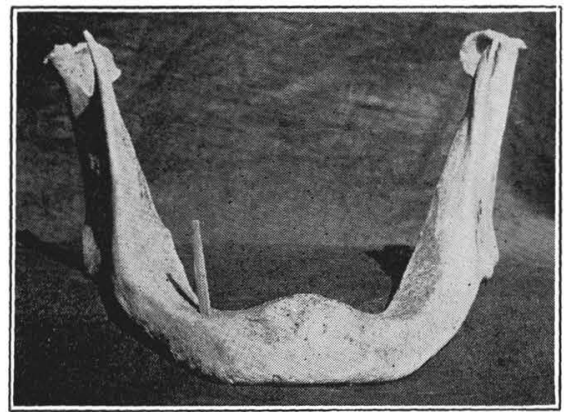

Figure 1.

touching the end of an applicator which has been touched in phenol to the spot, and a point $13 \mathrm{~mm}$. from the inferior border of the mandible should be marked with a pencil on the external or skin surface and the distance measured with dividers. (Note. An allowance of from $2 \mathrm{~mm}$. to $4 \mathrm{~mm}$. should be made by the operator for the covering of the mandible.) A guard should be placed on the needle at a corresponding distance, to avoid making too deep an injection.

Then starting the needle at the marked point and keeping the bevel of the needle in close contact with the bone and proceeding carefully, the needle will fall within the foramen, somewhere between the point of injection and the point $13 \mathrm{~mm}$. above the inferior border of the mandible.
When the guard reaches the point of injection then the needle is $13 \mathrm{~mm}$. from the inferior border of the mandible.

In $46 \frac{1}{2} \%$ of the cases the needle will be within the foramen when it has reached that point, and in $88 \%$ it will be found within the limits of 13 and 15 mm.

The mental foramen cannot always be located in the line slightly anterior to the apex of the second bicuspid. It varies and may be distal to the second bicuspid, but it will be found slightly anterior in at least $90 \%$ of the cases. Attention is also drawn to the fact, and

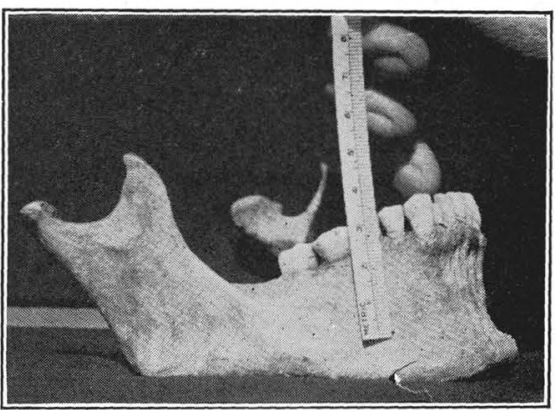

Figure 2.

it should be borne in mind that the window of the mental foramen in $89 \%$ of the cases, opens slightly to the distal (varying up to 300 ), $8 \%$ opens laterally, and only $3 \%$ open anteriorly. Therefore, to penetrate it, the needle should come from above (at a right angle to the mandible) and not at an angle from the mesial.

The measurements and observations, relative to the mandibular sulcus, are very interesting. The mandibular sulcus is the name given to the depression in the inner surface of the mandible immediately adjacent to the inferior alveolar foramen, and described as one centimeter in diameter. The inferior alveolar nerve occupies the center of the sulcus.

It is conceded that to produce man- 
dibular anesthesia, the anesthetic should be deposited within the sulcus, and that the best results are obtained when the anesthetic is deposited at the center of the sulcus.

One making a study of a large number

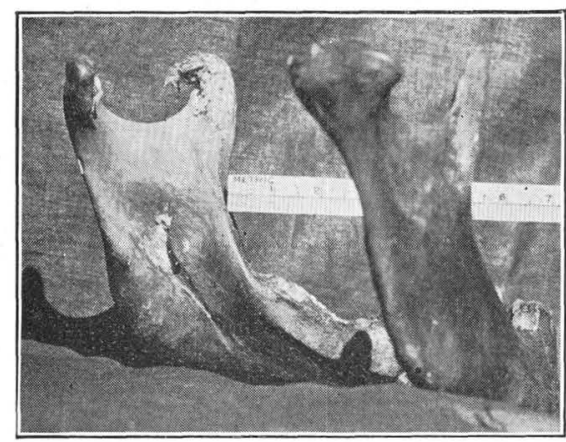

Figure 3 .

of mandibles is struck with the wide variations.

In the discussion of these points, the findings of a series of three measurements will be used.

(1) From the external oblique line to the internal oblique line.

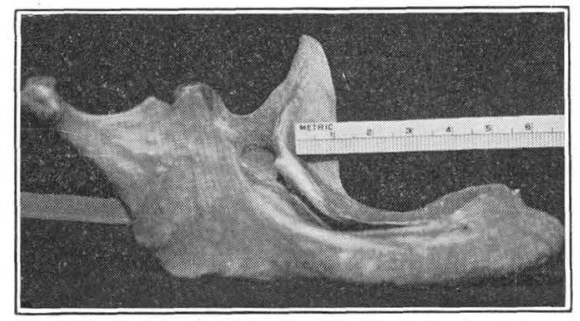

Figure 4.

(2) From the external oblique line to the center of the sulcus.

(3) From the external oblique line to the posterior border of the ramus.

The measurements for the first were made by laying a straight edge across the two external oblique lines and measuring with a $\mathrm{mm}$. ruler projecting at right angles from the straight edge, to the internal oblique line. This distance is very inconstant, ranging from $1 \mathrm{~mm}$. (Figure 3) to $11 \mathrm{~mm}$. (Figure 4).

It was also observed that the distance from the internal oblique line to the center of the sulcus is extremely variable, in some instances the internal oblique line practically ending in the lingula. (Figure 4.)

The extreme inconstancy of the internal oblique line has led the writer to the opinion that it (the internal oblique line) should be eliminated as a landmark in the technic for mandibular conductive anesthesia, except to palpate for passing the needle by it.

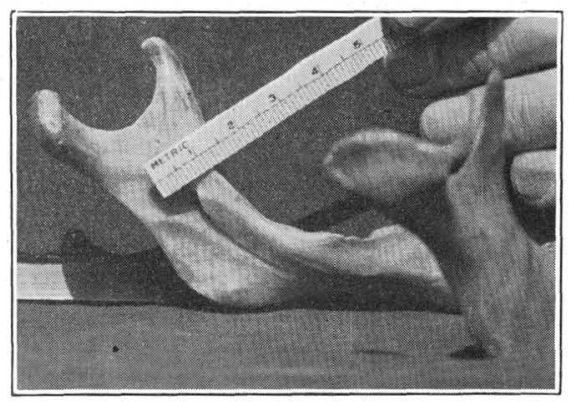

Figure 5.

2. The measurements from the external oblique line to the center of the sulcus, were made by placing the points of the dividers in those respective locations and measuring the spread with a mm. ruler. The charts show that this distance is also extremely inconstant, ranging from $13 \mathrm{~mm}$. (Figure 5) to 27 $\mathrm{mm}$. (Figure 6) with all the variations between.

3. The measurements from the external oblique line to the posterior border of the ramus, were made in a similar manner, with the dividers and the $\mathrm{mm}$. ruler, and the distance is variable in the same ratio, ranging from $20 \mathrm{~mm}$. (Figure 7 ) to $41 \mathrm{~mm}$. (Figure 8).

The reader will note that in these two 
widely varying cases the center of the sulcus falls practically two-thirds of the distance from the external oblique line to the posterior border of the ramus.

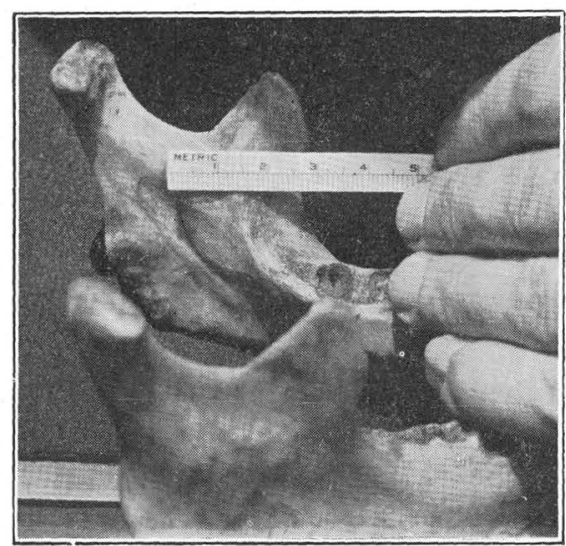

Figure 6.

The 200 cases measured, and all others which the writer has been able to observe, have shown that a point two-thirds the

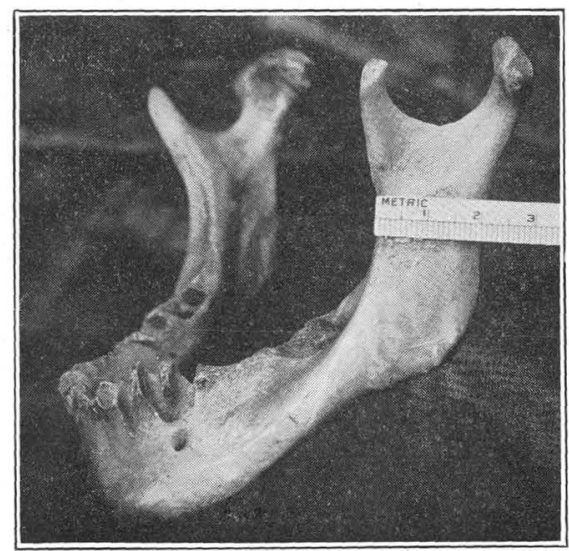

Figure 7 .

distance from the external oblique line to the posterior border of the ramus, falls within the width of the nerve of the center of the sulcus. This circumstance is constant, and establishes one of the most important facts in the techric for mandibular conductive anesthesia.

The writer believes that with the large number of measurements and observations made, the very important fact is established that it is possible to approximate the inferior alveolar nerve within the sulcus in every case of mandibular conductive anesthesia, by measuring the width of the ramus, with a ramometer. (Figure 9) and taking two-thirds of the distance as the depth of the injection.

In making this injection the needle should have a guard* (Figure 10) fixed at the proper distance, and the guard should be projected to the nucous

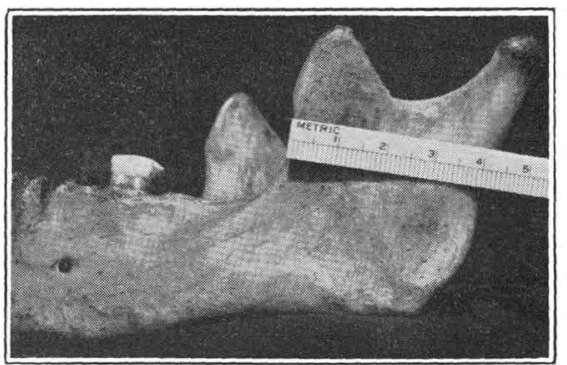

Figure 8.

fold, which will complete a triangle with the external oblique line and the center of the sulcus.

But in no case should the needle be projected more than $5 \mathrm{~mm}$. beyond the measured depth, because of the danger of injecting into the parotid gland. The parotid gland enfolds the posterior border of the ramus, and with the narrow rami (as narrow as $2 \mathrm{~cm}$.) there is great danger of this distance being exceeded, and the parotid gland injected. Also the posterior facial vein traverses the parotid gland at the posterior border of

*A small piece of vulcanite rubber can be used as a guard. This will not dissolve in alcohol. so it can be kept sterile. The guard (piece of vulcanite) can be seen at the end of the glass tube into which the needle is projecting. The tube is calibrated in $\mathrm{mm}$. for a distance of $5 \mathrm{~cm}$. and is used to drive the guard to the proper place on the needle. 
the ramus, (Sobatta-McMurrich), and is very easily penetrated, and it is the writer's opinion, that it is the injection of Procaine into this vein and its transmission by the blood stream to the brain, thereby producing a stimulation of the Medulla (vaso-motor center) producing a dilation of the abdominal blood vessels which causes a blood stasis. Not enough blood would be traveling to the brain to more than $2 \mathrm{~cm}$. from the external oblique line, and 76 were less than 2 $\mathrm{cm}$; or 150 in 200 , $(75 \%)$, would not be reached in the prescribed $2 \mathrm{~cm}$. injection. (Figure 9).

The ramometer consists of two parts, A. and B. A. slides thru the tube C. and is calibrated in $\mathrm{mm}$. for a distance of $5 \mathrm{~cm}$. C' stands at $\mathrm{O}$. when the lines D. and E. fall in the same plane. B.

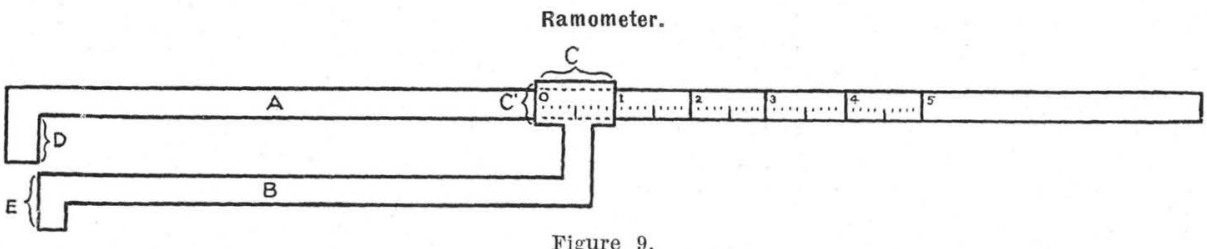

keep it supplied with oxygen, causing an anemia of the brain which is the prime cause for the synocope, dyspnoea, cyanosis and other unpleasant sequela, which have been more common and profound in mandibular anesthesia than in any other.

In all the literature on mandibular conductive anesthesia which the writer has been able to obtain, he has observed that from $1.5 \mathrm{~cm}$. to $2 \mathrm{~cm}$. have been designated as the depth of the injection.

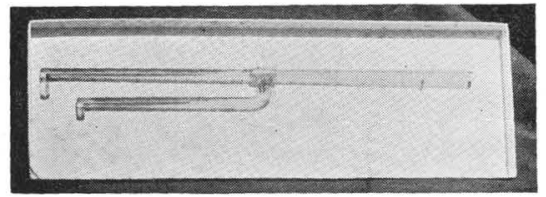

Figure 9-A.

Attention is here drawn to the wide variations in mandibles, and the reader will readily understand that it is unscientific to use any set distance as the depth of the injection and that in every case of mandibular conductive anesthesia a di[ferent depth of injection is required to reach the center of the sulcus, and that this distance should be determined in each case. Of the 200 sulci measured, in 74 cases the centers of the sulci were goes inside the check and E. rests on the external oblique line. A. goes outside the check, and D. hooks over the posterior border of the ramus and the width of the ramus is read at $C^{\prime}$ the end of the tube $\mathrm{C}$.

The next point to which the reader's attention is drawn is the method of determining the point of injection that will place the needle in the perpendicular plane of the mandibular sulcus. The instructions have been that the point of

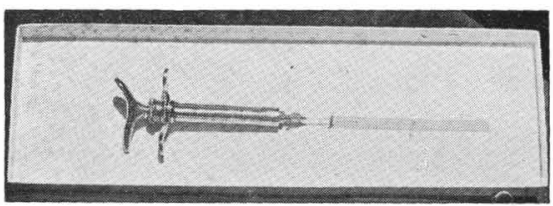

Figure 10.

injection should be $1 \mathrm{~cm}$. above the plane of the teeth or $1 \mathrm{~cm}$. above the last tooth. The extreme variations in all proportions in mandibles, as the reader will note even in the few figures herein given, will again readily convince one of the inaccuracy of any set distance.

The writer has several mandibles in which the point of injection should be as low as the line of the plane of the teeth, and some in which the center of 
the sulcus, (the proper point for depositing the anesthesia is $5 \mathrm{~mm}$. below the line of the plane of the teeth, therefore requiring the point of injection to be 5 $\mathrm{mm}$. below the plane of the teeth.) (Figure 11).

The writer also has mandibles in

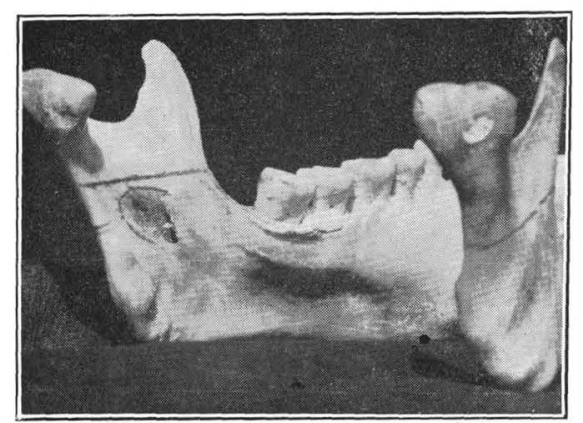

Figure 11.

which, if the prescribed point of injection (1 cm. above the plane of the teeth) is used, the needle will pass thru the sigmoid notch (Figure 12) or puncture the temporo-mandibular capsule.

Furthermore, there have been no in-

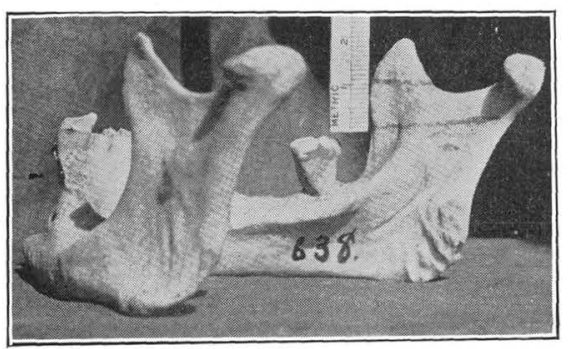

Figure 12.

structions to determine the point of injection in mandibles in which the posterior teeth were missing nor in edentulous mandibles. (Figures 1 and 5).

The writer has developed a technic for determining the perpendicular point of injection which is very simple and accurate. The reader will observe (Fig- ure 13) that in the external oblique line of each ramus there is a distinct notch. Different types of mandibles, the rami of which are from the widest to the narrowest, and from the longest to the shortest, are seen in this picture, and in every one the notch is plain.

This notch is constant in all mandibles which the writer has been able to observe. In the mouth this notch is very easily palpated because the temporal ligament has its attachment at the superior bulge of the notch, therefore amplifying it.

This notch is the one important landmark for determining the perpendicular point of injection for mandibular anesthesia. The operator should stand in front of the patient and palpate the

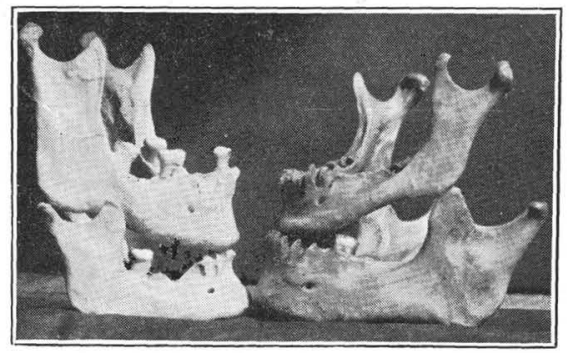

Figure 13.

notch with the thumb. The thumb is then turned inward at a right angle to the ramus, to the internal oblique line. These instructions should be carefully observed, as the needle should always travel at a right angle to the ramus.

If this technic is followed carefully the center of the thumb will point directly toward the sulcus, and therefore indicates the point and line of injection. This point may fall below the plane of the teeth, (Figure 11) or it may be more than $1 \mathrm{~cm}$. above $(1.7 \mathrm{~cm}$. in Figure 14).

This technic has proven to be accurate, in every case the writer has been able to observe (about $400 \mathrm{rami}$ ).

The perpendicular location of the 
sulcus is of extreme importance. When the regional anatomy is understood and the course of the inferior alveolar and lingual nerves is considered, then its full importance is appreciated. The inferior alveolar and lingual nerves descend from their bifurcation (slightly below the foramen ovale) downward along the medial side of the external pterygoid muscle, then turn outward and reach the ramus at a right angle, coming between the internal and external pterygoid muscles. Therefore the inferior alveolar nerve cannot be approximated for mandi-

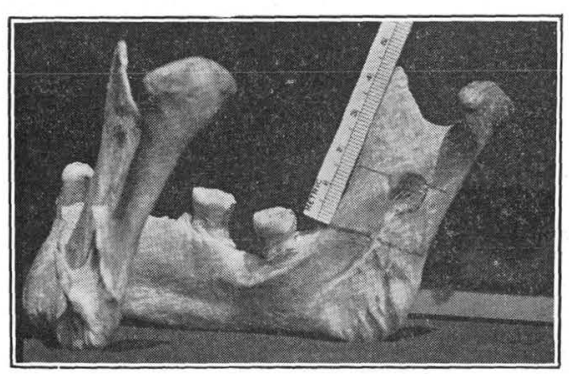

Figure 14.

bular anesthesia at any point above or below the mandibular sulcus.

The reader can readily understand then, that to reach the nerve and secure the desired anesthesia with the least amount of anesthetic, it must be deposited within the sulcus otherwise the anesthetist must depend upon a diluted anesthetic reaching the nerve by diffusion thru the tissues, and resulting in a partial, slow or no anesthesia, depending upon the distance from the sulcus at which the anesthetic was deposited.

The practical results obtained by following this technic carefully justify the writer in saying that there are no unseemly results from the injection of procaine for conductive anssthesia if it is done properly and that we are getting complete mandibular anesthesia, not in 15 to 25 minutes but in three minutes.
The measurements relative to the infraorbital foramen were made by placing one end of the dividers on the infraorbital ridge and the other end on the superior border of the formen, and measuring the spread on a mm. ruler. The charts of the 200 foramina measured, show this distance to vary from $2 \mathrm{~mm}$. to $12 \mathrm{~mm}$. In the skull on which the 2 $\mathrm{mm}$. distance was found, there were two infraorbital foramina on the left side measuring $2 \mathrm{~mm}$. and $3 \mathrm{~mm}$. respectively from the superior border of the infraorbital ridge; and in only one other skull was there a $3 \mathrm{~mm}$. distance found. Following is a table of the measurements from the infraorbital ridge to the infraorbital foramin.

One was 2 and $3 \mathrm{~mm}$. respectively.

One was $3 \mathrm{~mm}$.

Thirteen were $4 \mathrm{~mm}$.

Thirty-four were $5 \mathrm{~mm}$.

Forty-seven were $6 \mathrm{~mm}$.

Fifty were $7 \mathrm{~mm}$.

Twenty-seven were $8 \mathrm{~mm}$.

Eleven were $9 \mathrm{~mm}$.

Ten were $10 \mathrm{~mm}$.

Four were $11 \mathrm{~mm}$.

Two were $12 \mathrm{~mm}$.

The table shows that by allowing 3 $\mathrm{mm}$. as the diameter of the infraorbital foramen, and making the injection at the reflex of the mucous membrane in the bicuspid region, and projecting to the foramen, the foramen will have been found in $99 \%$ of the cases somewhere in its course when the needle has reached a point $7 \mathrm{~mm}$. below the superior border of the infraorbital ridge and in 144 cases of the 200 it will be at the foramen, when it has reached that point.

The infraorbital foramen is located at about the middle of the inferior border of the orbit, and opens in a depression beneath the infraorbital ridge. The window of the foramen opens at an angle of about $45^{\circ}$ mesially and downward 
(Figure 15) or toward the ala of the nose. To make an infraorbital conductive anesthesia the foramen should be palpated and the point marked with a pencil. If impossible to palpate, a point $7 \mathrm{~mm}$. below the middle of the infraorbital ridge should be marked with a pencil. The point of injection should be at the reflex of the mucous membrane between the first and second bicuspid, and this point should be marked with an applicator which has been touched in phenol. The distance between the two points should be measured and a guard

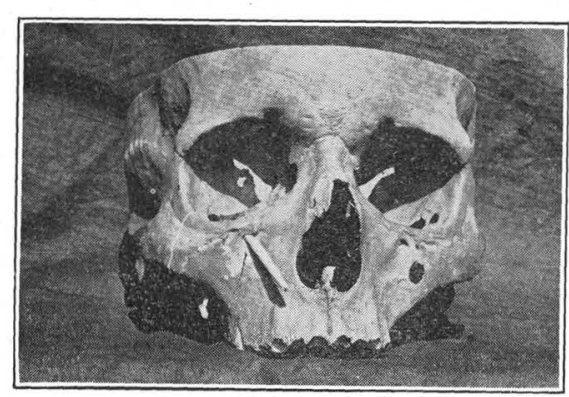

Figure 15.

placed on the needle at the proper position. The needle is then projected from the marked spot toward the infraorbital foramen, keeping the needle lightly in contact with the bone, and 1 c.c. of the anesthetic deposited as the needle proceeds and 1 c.c. should be deposited when the guard has reached the point of injection, which would place the enesthetic at the infraorbital foramen. Then the point of deposition should be carefully massaged to carry the anesthetic thru the anterior wall of the antrum and into the foramen where the anterior superior alveolar nerve is given off from the infraorbital nerve.

The point of injection to reach the posterior superior alveolar nerve and foramen for conductive anesthesia, is at the reflex of the mucous membrane $1 \mathrm{~cm}$. posterior to the malar process. The measurements for this foramen were made by placing one end of the dividers at the foramen and the other end at a point $1 \mathrm{~cm}$. posterior to the malar process and in a line drawn horizontally from the inferior point of the malar process (the malar process is palpable usually above the first malar).

This would be at the reflex of the mucous membrane.

The charts show the distance from this point to the posterior alveolar foramen to vary from $7 \mathrm{~mm}$. to $20 \mathrm{~mm}$. and that $89 \%$ of the foramina fall within the limit of $15 \mathrm{~mm}$. from the designated point.

There is no method of determining the exact location of the posterior superior alveolar foramen but it is the opinion of the writer that an injection $15 \mathrm{~mm}$. in depth, in this region, is sufficient to produce tuberosity conductive anesthesia. In some cases an injection just thru the attachment of the buccinator muscle would be sufficient, but it is impossible to locate the point exactly, therefore the $15 \mathrm{~mm}$. injection is recommended.

The following technic is advised. Locate a point at the reflex of the mucous membrane $1 \mathrm{~cm}$. posterior to the malar process. Insert the needle at this point and project it at an angle of $45^{\circ}$ up and back keeping the bevel of the needle close to the bone for a distance of $15 \mathrm{~mm}$.

One-half c.c. of a $2 \%$ procaine solution should be injected as the needle proceeds, and the remainder of 1 c. c. (onehalf c.c.) should be deposited when the $15 \mathrm{~mm}$. depth is reached. This would produce dental anesthesia, in the whole molar region, unless there was an innervation in the first molar region, from an anastemosis from the middle superior alveolar nerve in which case anesthesia could be produced by an infiltration in canine fossa.

For surgical anesthesia in the molar region another injection at the posterior palatine foramen is necessary. 


\section{ZYGOMATIC.}

The measurements for the zygomatic or spheno-maxillary injection were made with the same instruments and in a similar manner as heretofore described, and again the wide variations will not permit of a set distance being prescribed for the depth of the injection.

Complete dental maxillary anesthesia can be produced by depositing the anesthetic at the entrance of the maxillary nerve into the infraorbital canal, (in the spheno-maxillary fossa). This point is the most convenient and most practical for injecting for complete dental maxillary anesthesia.

The depth of this injection can be determined by comparative measurements. First, the point of entrance of the infraorbital nerve into the infraorbital canal, is directly posterior to the infraurbital foramen, and the nerve travels in practically a straight line in its course across the floor of the orbit. If this line is bisected at right angles by a plane, that plane will pass thru a point at the reflex of the mucous membrane $1 \mathrm{~cm}$. posterior to the molar process. Since this plane bisects the line, then any point on the plane is equidistant from its two ends (the posterior end of the infraorbital canal and the infraorbital foramen), therefore the distance from the point of injection $(1 \mathrm{~cm}$. posterior to the molar process) to the infraorbital foramen is equal to the distance from the point of injection to the posterior end of the infraorbital canal (the proper point for depositing the anesthetic) and is therefore the proper depth for the injection.

To make this injection the curved hui of the Fischer type syringe should be used. To produce maxillary conductive anesthesia the point of injection should be determined as described and marked. The distance from this point to the infraorbital foramen would be the proper depth to make the injection. This distance should be determined with calipers and measured and a guard placed on the needle at the proper position. The injection is then made and the needle kept lightly in contact with the bone, and projected in the plane described by the point of injection and the line from the posterior end of infraorbital canal to the infraorbital foramen, until the guard reaches the point of injection (1 c.c. of $2 \%$ procain solution produces complete maxillary anesthesia.)

These injections should be made only with a full knowledge of the anatomy of the region and the observance of sur.. gical asepsis in all that it means. 